\title{
A BLIND SYNCHRONOUS MULTI-USER DS-CDMA EQUALISER WITH PARTIAL LOADING
}

\author{
Mahmoud Hadef and Stephan Weiss \\ School of Electronics \& Computer Science \\ University of Southampton \\ Southampton SO17 1BJ, UK \\ \{m.hadef,s.weiss\}@ecs.soton.ac.uk
}

\begin{abstract}
In this paper, we propose a blind multiuser detection scheme in a direct sequence CDMA downlink scenario by means of a chiplevel equaliser, which can be updated even if not all possible users are active, i.e. the system is partially loaded. The active synchronous users are separated by re-establishing orthogonality of their spreading sequences in a common chip-level equaliser. The adaptation algorithm is mainly based on a constant modulus (CM) criterion applied to the active users. The inactive codes in the system must be considered, for which we proposed and compare three different methods: (i) a mean square error criterion for absent users, and a CM approach with (ii) zero modulus or (iii) the transmission of arbitrary signals with small code amplitude. For all three cases, stochastic gradient descent algorithms are derived. The proposed algorithms are analysed and compared through various simulations, which demonstrate the algorithms' convergence and BER performance.
\end{abstract}

\section{INTRODUCTION}

In direct sequence (DS) CDMA, the transmitted users are multiplexed by orthogonal codes. However, transmission over a dispersive channel destroys the mutual orthogonality of these codes, and as a result, the received and code-demultiplexed user signals are subject not only to inter-symbol interference (ISI) due to channel dispersion but also to multiple access interference (MAI) due to the loss of code orthogonality.

A popular approach to suppress MAI and ISI on a user is the minimum output power (MOE) algorithm blindly cancelling MAI and ISI terms but passing the desired user by code-constraints [1, 2], which is essentially Frost's linearly constrained minimum variance beamformer [3]. For the DS-CDMA downlink, the recovery of several synchronous users at the same time exploits more knowledge of the system. Non-blind multiuser schemes, such us using the mean squared error (MSE) criterion, in turn are based either on the knowledge of a pilot $[4,5]$ or training sequences [6]. Blind schemes have been performed using a constant modulus (CM) criterion $[7,8,9]$, whereby the derived algorithms either neglect the dispersiveness of the channel $[7,9]$ or spreading $[8,10]$, whereby the later additionally require mutual decorrelation of the recovered user sequences. In [11], a blind scheme similar to [8, 10] has been developed, whereby the despreading in the DS-CDMA receiver ensures the orthogonality of the recovered sequences, and a CM criterion on all users suffices. The algorithm in [11] is however only suitable for a fully loaded system, in which all possible users are active.
In this paper we address a blind chip-level equalisation scheme, but consider a partially loaded scenario, where not all users may be active. Based on the definition of a signal model in Sec. 2, two hybrid CM/MSE cost functions are derived in Sec. 3, which are suitable for a partial loaded DS-CDMA system. Sec. 4 presents stochastic gradient algorithms, which are structurally similar to a multiple error filtered-X LMS algorithm in [12] in feeding a code filtered equaliser input to the update algorithms. Simulations are presented in Sec. 5, and conclusions drawn in Sec. 6.

\section{SIGNAL MODEL}

We consider the DS-CDMA downlink model in Fig. 1 with a maximum of $N$ symbol-synchronous active users, which for simplicity are assumed to have the same rate. In the case of a partially loaded system with $K \leq N$, we assume the first $K$ users with signals $u_{l}[n], l=0$ (1) $\bar{K}-1$, to be active, while for the remaining $K-N$ user signals $u_{l}[n]=0, l=K(1) N-1$ and $\forall n$. The signals $u_{l}[n]$ are code multiplexed using Walsh sequences of length $N$ extracted from a Hadamard matrix $\mathbf{H}$. The resulting chip rate signal, running at $N$ times the symbol rate, is further scrambled by $c[m]$ prior to transmission over a channel with dispersive impulse response $g[\mathrm{~m}]$ and corruption by additive white Gaussian noise $v[m]$, which is assumed to be independent of the transmitted signal $s[m]$.

The dispersive channel $g[m]$ destroys the orthogonality of the Walsh codes, such that direct decoding of the received signal $r[\mathrm{~m}]$ with descrambling by $c^{*}[m]$ and code-matched filtering by $\mathbf{H}^{\mathrm{T}}$ will lead to MAI and ISI corruption of the decoded user signals $\hat{u}_{l}[n], l=0(1) N-1$. In order to re-establish orthogonality of the codes, a chip rate equaliser $w[\mathrm{~m}]$ can be utilised $[6,5]$. In the following, we are concerned with the blind updating of the equaliser coefficients $w[m]$.

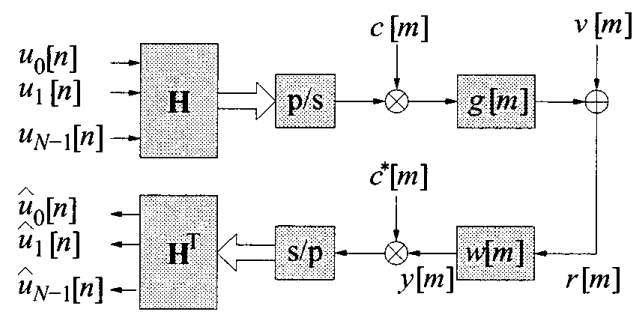

Fig. 1. DS-CDMA downlink signal model. 


\section{MULTIUSER EQUALISATION CRITERIA}

We first derive the detected user signals $\hat{u}_{l}[n]$ as a function of the chip-rate equaliser $w[m]$. Based on this, we state suitable cost functions based on which the equaliser can be adapted.

\subsection{Demultiplexed User Signals}

For the decoding, Walsh sequences are used as matched filters. The sequence for decoding the $l$ th user, contained in a vector $\mathbf{h}_{l}$, can be taken from an $N \times N$ Hadamard matrix,

$$
\mathbf{H}^{\mathrm{T}}=\left[\begin{array}{llll}
\mathbf{h}_{0} & \mathbf{h}_{1} & \cdots & \mathbf{h}_{N-1}
\end{array}\right]^{\mathrm{T}} .
$$

The $l$ th user is thus decoded as

$$
\begin{aligned}
& \hat{u}_{l}[n]=\mathbf{h}_{l}^{\mathrm{T}} \cdot\left[\begin{array}{cc}
c^{*}[n N] & \multicolumn{0}{c}{} \\
c^{*}[n N-1] & \\
& \ddots \\
\mathbf{0} & c^{*}[n N-N+1]
\end{array}\right] \cdot\left[\begin{array}{c}
y[n N] \\
y[n N-1] \\
\vdots \\
y[n N-N+1]
\end{array}\right]
\end{aligned}
$$

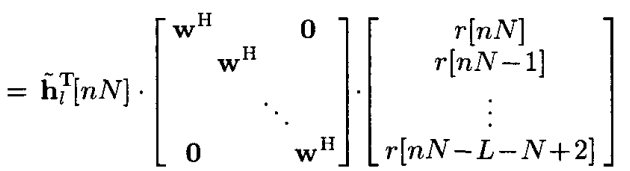

whereby the descrambling code $c^{*}[m]$ has been absorbed into a modified and now time-varying code vector $\tilde{\mathbf{h}}_{l}[n N]$, and $\mathbf{w} \in \mathbb{C}^{L}$ contains the equaliser's $L$ chip-spaced complex conjugate weights. Rearranging $\mathbf{w}$ and $\tilde{\mathbf{h}}_{l}[n N]$ yields

$$
\begin{aligned}
\hat{u}_{l}[n] & =\mathbf{w}^{\mathrm{H}} \cdot\left[\begin{array}{cc}
\tilde{\mathbf{h}}_{l}^{\mathrm{T}}[n N] & \mathbf{0} \\
& \tilde{\mathbf{h}}_{l}^{\mathrm{T}}[n N] \\
& \ddots \\
\mathbf{0} & \tilde{\mathbf{h}}_{l}^{\mathrm{T}}[n N]
\end{array}\right] \cdot\left[\begin{array}{c}
r[n N] \\
r[n N-1] \\
\vdots \\
r[n N-L-N+2]
\end{array}\right] \\
& =\mathbf{w}^{\mathrm{H}} \mathbf{H}_{l}[n N] \mathbf{r}_{n N},
\end{aligned}
$$

with $\mathbf{H}_{l}[n N] \in \mathbb{C}^{L \times(N+L-1)}$ being a convolutional matrix comprising of the $l$ th user's modified code vector $\tilde{\mathbf{h}}^{\mathrm{T}}[n]$ and $\mathbf{r}_{n N} \in$ $\mathbb{C}^{N+L-1}$.

\subsection{Cost Functions}

We assume that the $K$ active user signals $u_{l}[n]$ consist of symbols with a constant modulus $\gamma$, such as BPSK, QPSK, or 8-PAM. Therefore, the idea is to blindly adapt the equaliser and track any channel variations by forcing all received user symbols onto a constant modulus. In following, we discuss three possible cost functions which may be suitably minimised.

CM Algorithm. By allowing different moduli $\gamma_{l}$ for the various decoded signals $\hat{u}_{l}[n]$, we can set $\gamma_{l}=0$ for the remaining $N-K$ inactive users signals $u_{l}[n]=0$ for $l=K(1) N-1$. Thus a suitable cost function to be minimised by the equaliser coefficients can be formulated as $\xi_{\mathrm{CM}}$,

$$
\begin{aligned}
& \xi_{\mathrm{CM}}= \mathcal{E}\left\{\sum_{l=0}^{N-1}\left(\gamma_{l}^{2}-\left|\hat{u}_{l}[n]\right|^{2}\right)^{2}\right\}=\sum_{l=0}^{N-1} \xi_{\mathrm{CM}, l} \\
& \text { with } \quad \gamma_{l}= \begin{cases}\gamma, & l \in[0 ; K-1] \\
\tilde{\gamma}, & l \in[K ; N-1]\end{cases}
\end{aligned}
$$

which measures the deviation of each of the $N$ users' decoded symbols from the desired modulus. Note that $\mathcal{E}\{\cdot\}$ denotes the expectation operator and that the modulus $\tilde{\gamma}_{l}=0$ for inactive users. The the second term in (3) is important to be included, as otherwise the equalisation criterion is under-determined, and the correct signals would not necessarily be extracted in the despreading operation. The decoded signals $\left.\hat{u}_{[} n\right]$ of inactive users contain channel noise and an MAI term. The minimisation of (3) minimises the MAI on the inactive users and hence ensures that the overall system is fully determined.

CM Algorithm with Signal Injection. Alternative to setting $\tilde{\gamma}_{l}=$ 0 for inactive users, arbitrary constant modulus user signals with a finite $\tilde{\gamma}_{l} \ll \gamma$ may be injected at the transmitter. This permits to persistently excite the system during adaptation, particularly if the DS-CDMA downlink is sparsely loaded, although this has the disadvantage of somewhat increasing the transmitted signal power. A similar problem is however encountered in pilot-based adaptation schemes $[4,5]$.

CM/MSE Algorithm. Different from (3), driving the decoded inactive user signals to zero can also be accomplished in the mean squared error (MSE) sense, such that a combined CM/MSE cost function

$$
\begin{aligned}
\xi_{\mathrm{CM} / \mathrm{MSE}}= & \sum_{l=0}^{K-1} \xi_{\mathrm{CM}, l}+\sum_{l=K}^{N-1} \xi_{\mathrm{MSE}, l} \\
& \text { with } \quad \xi_{\mathrm{MSE}, l}=\mathcal{E}\left\{\left|\hat{u}_{l}[n]\right|^{2}\right\}
\end{aligned}
$$

arises.

The equaliser coefficients $\mathbf{w}$ can be determined such that the above cost functions are minimised. However, a manifold of solutions exist for an optimum,

$$
\mathbf{w}_{\mathrm{opt}}=\arg \min _{\mathbf{w}} \xi_{\mathrm{CM}} \quad \text { or } \quad \mathbf{w}_{\mathrm{opt}}=\arg \min _{\mathbf{w}} \xi_{\mathrm{CM} / \mathrm{MSE}}
$$

since an ambiguity with respect to a complex rotation $e^{j \varphi}, \varphi \in$ $[0 ; 2 \pi]$, cannot be resolved by any of the above criteria. This rotation invariance could be overcome by differential encoding or the transmission of a synchronisation word.

\section{STOCHASTIC GRADIENT BASED BLIND ADAPTATION}

Simple adaption rules for the equaliser can be obtained by considering stochastic gradient descent techniques, whereby an iterative update rules is utilised for the equaliser coefficient vector $\mathbf{w}_{n}$ at time $n$,

$$
\begin{aligned}
\mathbf{w}_{n+1}= & \mathbf{w}_{n}-\mu \nabla \hat{\xi} \\
& \text { with } \hat{\xi} \in\left\{\hat{\xi}_{\mathrm{CM}}, \hat{\xi}_{\mathrm{CM} / \mathrm{MSE}}\right\}
\end{aligned}
$$

where $\mu$ is the algorithm step size, and $\nabla$ the gradient operator applied to instantaneous cost functions $\hat{\xi}_{\mathrm{CM}}$ and $\hat{\xi}_{\mathrm{CM} / \mathrm{MSE}}$. These instantaneous estimates are obtained from (3) and (4) by dropping expectation operations, resulting in

$$
\hat{\xi}_{\mathrm{CM}}=\sum_{l=0}^{N-1} \hat{\xi}_{\mathrm{CM}, l}=\sum_{l=0}^{N-1}\left(\gamma_{l}^{2}-\left|\hat{u}_{l}[n]\right|^{2}\right)^{2}
$$


and

$$
\begin{aligned}
\hat{\xi}_{\mathrm{CM} / \mathrm{MSE}} & =\sum_{l=0}^{K-1} \hat{\xi}_{\mathrm{CM}, l}+\sum_{l=K}^{N-1} \hat{\xi}_{\mathrm{LMS}, l} \\
& =\sum_{l=0}^{K-1}\left(\gamma_{l}^{2}-\left|\hat{u}_{l}[n]\right|^{2}\right)^{2}+\sum_{l=K}^{N-1}\left|\hat{u}_{l}[n]\right|^{2}
\end{aligned}
$$

where the instantaneous MSE related expression is referred to as least mean squares (LMS) term. The gradient terms can be obtained for the CM components of the instantaneous cost functions as

$$
\begin{aligned}
\frac{\partial \hat{\xi}_{\mathrm{CM}, l}}{\partial \mathbf{w}^{*}} & =-2\left(\gamma_{l}^{2}-\left|\hat{u}_{l}[n]\right|^{2}\right) \frac{\partial}{\partial \mathbf{w}^{*}} \hat{u}_{l}[n] \hat{u}_{l}^{\mathrm{H}}[n] \\
& =-2\left(\gamma_{l}^{2}-\left|\hat{u}_{l}[n]\right|^{2}\right) \mathbf{H}_{l}[n N] \mathbf{r}_{n N} . \\
& =-2\left(\gamma_{l}^{2}-\left|\hat{u}_{l}[n]\right|^{2}\right) \mathbf{H}_{l}^{\mathrm{H}}[n N] \mathbf{w} \\
& \mathbf{H}_{l}[n N] \mathbf{r}_{n N} \hat{u}_{l}^{*}[n] .
\end{aligned}
$$

Similarly, the gradient components of the MSE part of the instantaneous cost function can be derived as

$$
\begin{aligned}
\frac{\partial \hat{\xi}_{\text {LMS }, l}}{\partial \mathbf{w}^{*}} & =\frac{\partial}{\partial \mathbf{w}^{*}}\left(\mathbf{w}^{\mathrm{H}} \mathbf{H}_{l}[n N] \mathbf{r}_{n N} \mathbf{r}_{n N}^{\mathrm{H}} \mathbf{H}_{l}^{\mathrm{H}}[n N] \mathbf{w}\right) \\
& =\mathbf{H}_{l}[n N] \mathbf{r}_{n N} \hat{u}_{l}^{*}[n] .
\end{aligned}
$$

This permits to assemble stochastic gradient descent algorithms according to (6) for the various cost functions derived in Sec. 3. The algorithms presented by (6) with its components in (9) and (10) differs from the standard CM algorithm [13] or its extension in [8] in the inclusion of a code filtered term $\mathbf{H}_{l}[n N] \mathbf{r}_{n N}$ rather than just the equaliser input $r[n]$. A similar structure has been derived in [12] for a purely least mean squares based criterion, and its general approach has been labelled as filtered error filter regressor scheme in the literature [14]. In the following, we refer to the proposed updating rules as filtered-R multiple error (FIRMER) CM or $\mathrm{CM} / \mathrm{LMS}$ algorithm.

\section{SIMULATION RESULTS}

The three stochastic gradient algorithms derived in Sec. 4 will be compared below for two channel impulse responses with mild and more sever dispersive characteristics as given in Fig. 2. We first demonstrate and compare the convergence behaviour in Sec. 5.1 and later the bit error performance in Sec. 5.2.
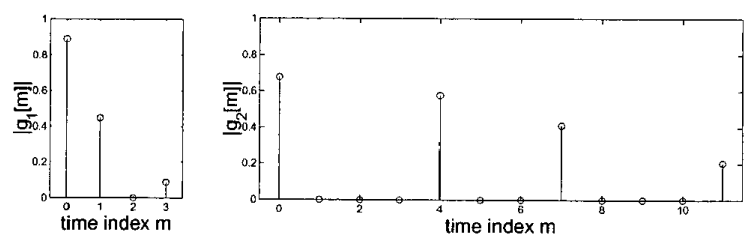

Fig. 2. Modulus of complex valued channel impulse responses $g_{1}[m]$ (left) and $g_{2}[m]$ (right).
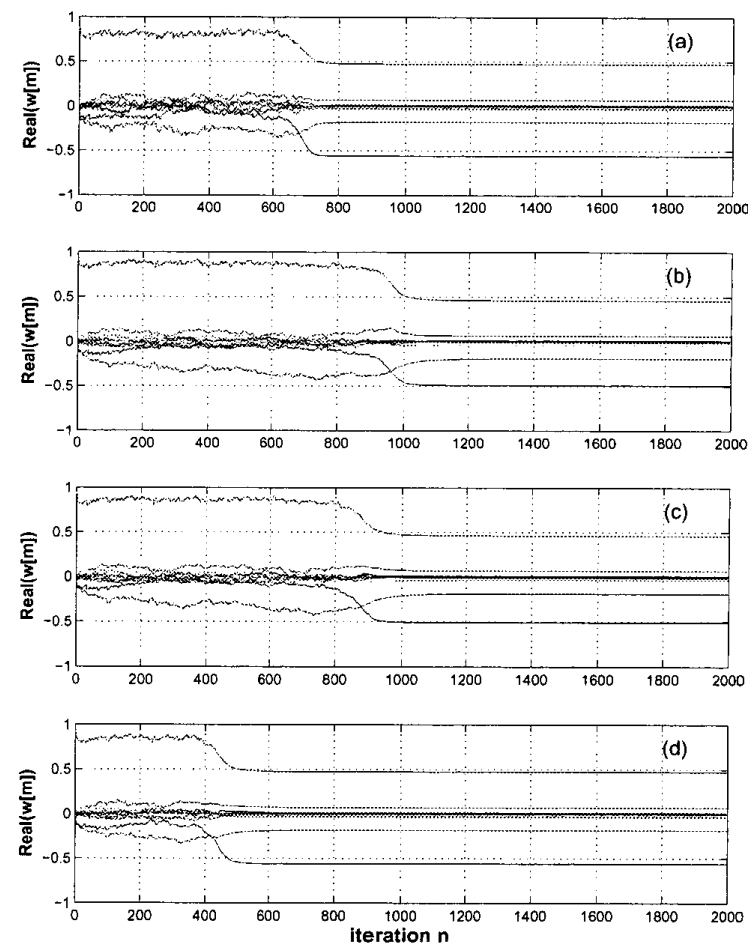

Fig. 3. Weight trajectories of the $N=K=16$ fully loaded system with FIRMER-CM algorithm, and of the partially loaded system with $N=16$ and $K=12$ for (b) the FIRMER-CM with $\tilde{\gamma}=0$ and (c) the FIRMER-CM with $\tilde{\gamma}=0.1$, as well as (d) of the combined FIRMER-CM/LMS algorithm.

\subsection{Convergence}

In order to demonstrate and compare the convergence behaviour of the proposed algorithms, we utilise an $N=16$ DS-CDMA downlink system to transmit $K=12$ active QPSK user signals over $g_{1}[m]$ in the absence of channel noise. We utilise the three proposed algorithms to update the chip-level equaliser with $L=10$ coefficients. The adaptation is initialised with the second coefficient in the weight vector set to unity. With the step size selected such as to obtain maximum convergence speed without incurring divergence, the evolution of the filter coefficients' real part of the three algorithms is shown in Fig. 3 compared to a $K=N=16$ fully loaded system. The learning curves in Fig. 3 are the instantaneous cost functions $\hat{\xi}_{\mathrm{CM}}$ in (7) and $\hat{\xi}_{\mathrm{CM} / \mathrm{MSE}}$ in (8) given in Fig. 4.

It can be noted from Figs. 3 and 4 that the algorithms succeed to minimise their cost functions, whereby a remaining error floor is due to model truncation. The injection of 4 QPSK signals with a small modulus for the inactive users can be seen to improve the convergence of the system somewhat compared to setting $\tilde{\gamma}=0$. In contrast however, the fully loaded system - which can be interpreted as a partially loaded system with $\tilde{\gamma}=\gamma$ ) - can attain a faster rate, i.e. the convergence rate increases with increasing modulus of the injected signals. The partially loaded FIRMERCM/LMS yet outperforms the fully loaded system. 


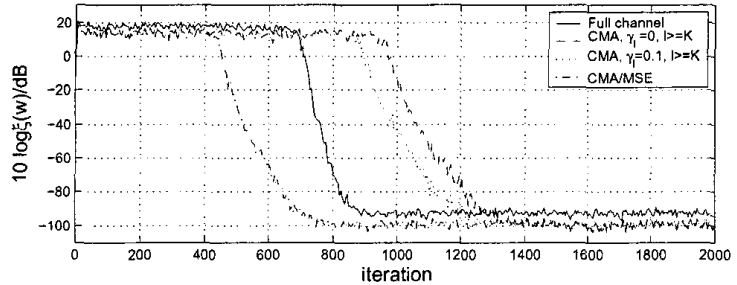

Fig. 4. Learning curves corresponding to the cases in Fig. 3.

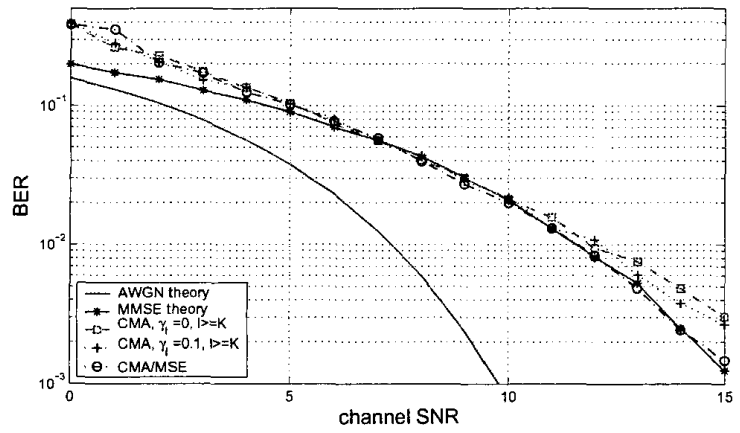

Fig. 5. BER performance of three proposed adaptation algorithms of the channel SNR compared to an analytical MMSE channel equaliser and the BER in an AWGN channel

\subsection{Bit Error Performance}

For $N=8$ users with $K=6$ active users, we have adapted the previous three algorithms under various SNR conditions for the channel impulse response $g_{2}[m]$ with $L=64$ equaliser coefficients. With the centre tap set to unity and an appropriately adjusted $\mu$, the algorithms have been given $10^{3}$ symbol periods in all cases to converge prior to correction of the phase rotation and bit error rate (BER) measurement. The BER results are given in Fig. 5 in comparison to the optimal QPSK performance in a dispersionfree AWGN channel and the analytical minimum MSE (MMSE) solution. Note that the three proposed algorithms show similar bit error performance and closely approach the MMSE performance, except for the FIRMER-CM cases at high SNR due to insufficient convergence, and at low SNR for all cases.

\section{CONCLUSIONS}

Blind equalisation approaches for a DS-CDMA downlink scenario with partial loading have been presented, which aim to enforce $\mathrm{CM}$ conditions on the various active user signals and either $\mathrm{CM}$ or MSE criteria on the remaining inactive users. Stochastic gradient algorithms have been derived, which differ from previously $\mathrm{CM}$ algorithms by a code-prefiltering of its input and require no additional constraints. For the inactive user part of the system, the simulations suggest that a $\mathrm{CM}$ criterion, even if additional signals are injected into the system, are inferior to the introduction of an MSE cost function. The later has the additional advantage of a lower implementational cost and does not increase the power of the transmitted signal.

\section{REFERENCES}

[1] M. Honig, U. Madhow, and S. Verdu, "Blind Adaptive Multiuser Detection," IEEE Transactions on Information Theory, vol. 41, no. 4, pp. 944-960, July 1995.

[2] R. Schober, W. H. Gerstacker, and L. H. J. Lampe, "Comparison of MOE and blind LMS," IEEE Communications Letters, vol. 7, no. 5, pp. 204-206, May 2003.

[3] O. L. Frost, III, "An Algorithm for Linearly Constrained Adaptive Array Processing," Proceedings of the IEEE, vol. 60, no. 8, pp. 926-935, August 1972.

[4] F. Petre, M. Moonen, M. Engels, B. Gyselinckx, and H.D. Man, "Pilot-Aided Adaptive CHip Rate Equalizer Receiver for Interference Suppression in DS-CDMA Forward Link," in IEEE Vehicular Technology Conference, Sept. 2000, vol. 1, pp. 303-308

[5] P. Schniter and A. R. Margetts, "Adaptive Chip-Rate Equalization of Downlink Multirate Wideband CDMA," in Asilomar Conference on Signals, Systems, and Computers, Monterey, CA, November 2002, pp. 1228-1232.

[6] I. Stirling, D. Garcia-Alis, S. Weiss, G. Rice, and R.W. Stewart, "A Joint Adaptive MMSE Downlink Receiver," in Asilomar Conference on Signals, Systems, and Computers, Monterey, CA, November 2000.

[7] K. Fukawa and H. Suzuki, "Orthogonalizing Matched Filter (OMF) Detection for DS-CDMA Mobile Radio Systems," in IEEE Global Telecommunications Conference, 1994, vol. 1, pp. 385-389.

[8] C. Papadias and A. Paulraj, "A Constant Modulus Algorithm for Multiuser Signal Separation in Presence of Delay Spread Using Antenna Arrays," IEEE Signal Processing Letters, vol. 4, no. 6, pp. 178-181, June 1997.

[9] Y. Huang and P. M. Djurić, "Multiuser Detection of Synchronous Code-Division Multiple-Access Signals by Perfect Sampling," IEEE Transactions on Signal Processing, vol. 7, no. 50, pp. 1724-1734, July 2002.

[10] S. Lambotharan, J. A. Chambers, and A. G. Constantinides, "Adaptive Blind Retrieval Techniques for Multi-User DSCDMA Signals," IEE Electronics Letters, vol. 35, no. 9, pp. 693-695, April 1999.

[11] S. Weiss and M. Hadef, "Blind Chip-Rate Multiuser Equalisation," in IEE Colloquium on DSP enabled Radio, Livingston, Scotland, September 2003, pp. 436-443.

[12] S.J. Elliott, I.M. Stothers, and P.A. Nelson, "A Multiple Error LMS Algorithm and its Application to the Active Control of Sound and Vibration," IEEE Transactions on Acoustics, Speech, and Signal Processing, vol. 35, no. 10, pp. 1423 1434, October 1987

[13] C. R. Johnson, P. Schniter, T. J. Endres, J. D. Behm, D. R. Brown, and R. A. Casas, "Blind Equalization Using the Constant Modulus Criterion: A Review," Proceedings of the IEEE, vol. 86, no. 10, pp. 1927-1950, October 1998

[14] J. R. Treichler, R. J. Johnson, and M. G. Larimore, Theory and Design of Adaptive Filters, Prentice Hall, 2001. 